\title{
High Efficiency Cooling and Heating Channels for Injection Moulding FREITAS Paulo ${ }^{1,2, a}$, SANTOS Cyrili1,3,b, CARREIRA Pedro ${ }^{1,3, c}$ and MATEUS Artur ${ }^{1,2, d}$
}

\author{
${ }^{1}$ CDRsp - Centre for rapid and sustainable product development, Rua de Portugal - Zona \\ Industrial, 2430-028 Marinha Grande, Portugal \\ ${ }^{2}$ ESTG - Escola Superior de Tecnologia e Gestão, Campus 2 - Morro do Lena, Alto do Vieiro, \\ Apt 4163, 2411-901 Leiria, Portugal \\ ${ }^{3}$ CEMMPRE - Centre for Mechanical Engineering, Materials and Processes, Paço das Escolas, \\ 3004-531 Coimbra, Portugal \\ apaulo.freitas@ipleiria.pt, bcyril.santos@ipleiria.pt, cpedro.s.carreira@ipleiria.pt, \\ dartur.mateus@ipleiria.pt
}

Keywords: Mould conformal cooling, Channel fins; High aspect ratio; Topological optimization; Mould design methodology.

\begin{abstract}
For many years traditional injection moulding has been the norm for obtaining polymer based products, and yet this cycle still shows potential for improvement. This paper will encompass the potential in the thermal part of the cycle, by introducing a novel approach to conventional tubular cooling designs, empowered by additive technologies this reiterates what's being known as conformal cooling.

Different geometries and techniques are compared to determine the optimal cross section layout of the fluid channels and inner surface of the moulding parts. If the cooling achieved is sufficiently fast the crystallographic growth of the material can be manipulated to obtain specific properties observable in the mesoscale. This however can be transversely applied in other heat exchanging structures in future studies.
\end{abstract}

\section{Introduction}

The injection moulding process is the preferred method to produce plastic parts, it allows the automated production of complex geometries at high production rates [1].

Developments have been made to improve part quality, like the positioning of the injection point, parting line pressures and temperature and to shorten the process time with the mechanisms used for the positioning, clamping and extraction of the part, by using electrical actuation systems instead of hydraulic [2].

However, these developments do not account for the impact of the moulding surface temperature in the part finish, neither the cooling time required for the polymer crystallization and to reduce warpage after extraction, which can amount to $80 \%$ of the total process time $[3,4]$.

The occurrences described come from the lack of thermal management in the mould space, usually an ambient temperature moulding surface is met with a "searing" flow of polymer that crystallizes as soon as heat is transferred between surfaces. This early crystallization dictates the growth orientation of the spherulites which isn't always favorable [5,6], because engineers often project a part envisioning an orthotropic behavior from the material or design with a higher safety coefficient, this makes for either unpredictable or heavier parts.

As for the warpage upon the extraction, it's due to unevenness of the moulding surfaces' thermal profile upon release [6]. This thermal profile is affected by the initial conditions like the polymer's injection that heats the mould in a dissimilar manner and the cooling when is far from ideal also contributes to this unevenness.

To understand what has been done before to tackle the challenges in this field there will be a brief state of the art analysis that is going to establish what the goal of each strategy was, what enabled it 
and what still hinders it. Following this path could help realize what advancements can be made using additive technologies and which scientific principles can be applied in the generation of structures.

Subsequently several geometries for cooling channels, some conventional and others with higher aspect ratio, are going to be analyzed with computer aided engineering [CAE] tools to determine which ones could best implemented and optimized in a larger scale.

\section{Industry proven techniques}

The common practice for when cooling is desired, is to make straight drilled channels which then may form complex structures if end caps and plugs are used. This solution is adequate to shorten the cycle's duration, but due to the complexity that the wanted part might have, it might produce hot spots that cause warpage when the releasing the mould [4], since the channels created recurring subtractive manufacturing.

Another solution is the insertion of bubblers or baffles, as illustrated in Figure 1 for when space is limited, these provide a way for the coolant to circulate within the mould insert. But for when this is not possible conductive pins or isobars [7] are used to convey the heat to an external cooling application.
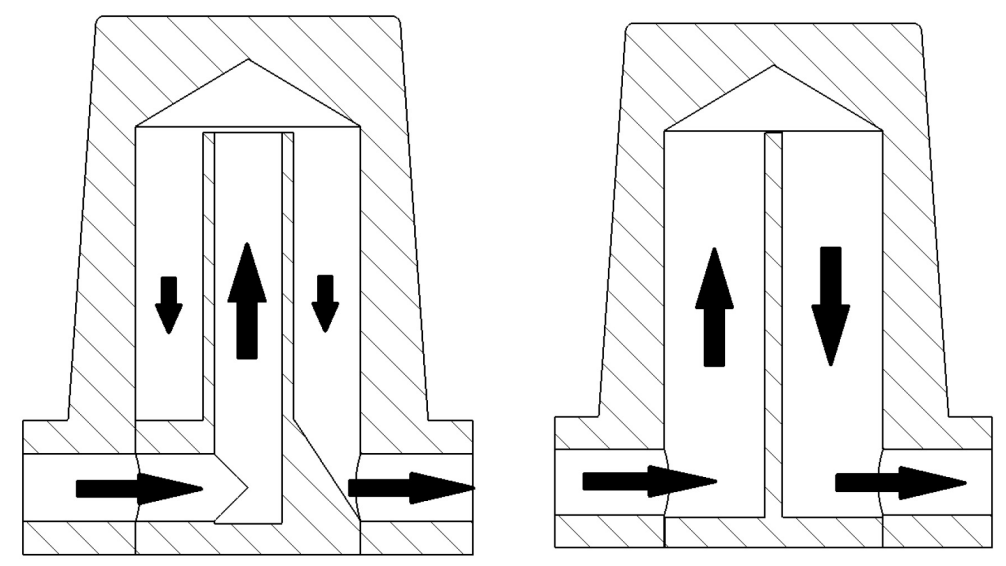

Figure 1-Examples of application of bubblers and baffles.

In recent years it has been common place to solve these issues with the use of conformal cooling, the concept is no longer novelty, but the advantages are clear, cooling channels that are designed to closely follow the shape of the moulding surface, like the ones found in Fig. 2, can prevent hot spots and reduce the cycle's overall time [4]. Due to the complexity of these passages additive manufacturing methods are best suited, however those technologies aren't being exploited to their fullest potential.
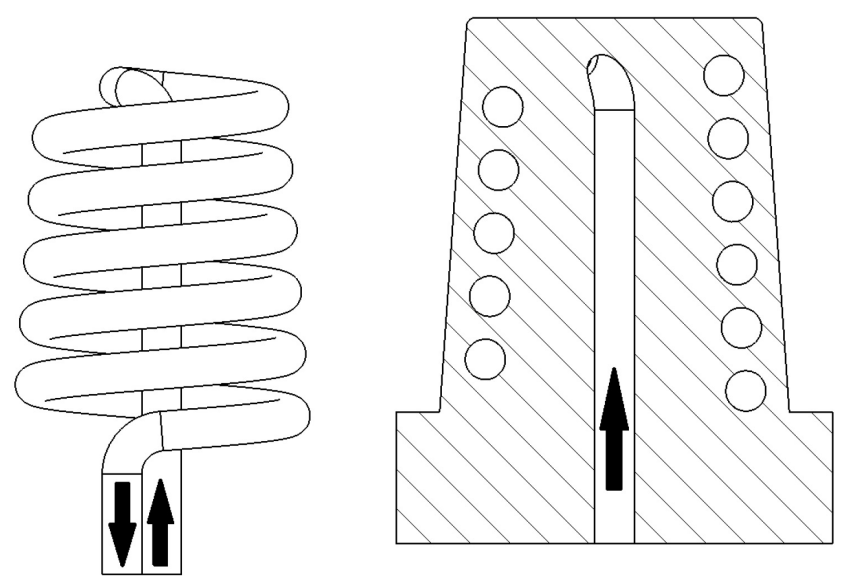

Figure 2- Examples of application of conformal cooling channels and cross section. 
Different constructal techniques are being developed to take advantage of additive manufacturing processes' potential. Instead of using round channels that envelop the surface topological optimizations are being considered [8], nevertheless this approach requires sophisticated computational tools and mathematical models that are not "calibrated" for volumes with the target of both minimizing the time for the temperature to reach a certain threshold and to have lowest thermal gradient on a surface.

\section{A different take}

Given the current limitations of the topological methods and the simplicity of most empiric designs comes a different approach, taking conventional channels or cavities and populate them with fins. Following the principles of high aspect ratio cooling, these fins can take the form of slots within the passage or radial fins, many other configurations can be had.

This solution could solve both the problems mentioned above by permitting the utilization of the channels for cooling and heating. If the mould is heated as soon as the parts are ejected, it could create an even surface temperature for the beginning of the injection, nullifying the issue of premature crystallization as the surface temperature matches the polymers' [5]. On the second front such solution could offer low inertia to temperature changes and be more effective mean to remove the unwanted heat, thus reducing the cycle's time.

This papers will confront traditional geometries like circular and square profiles [Fig. $3 \& 4$ ], against high aspect ratio designs by introducing inside cooling channels. Two form factors will be considered $4.6 \mathrm{~mm}$ and $15.8 \mathrm{~mm}$, these are not nominal integer values in order to facilitate the implementation of the finned structures that will be discussed later.

On this analysis the following geometries will be examined, of which squared ducts in Figure 3 are represented in the 4.6 millimeters form factor. Also represented only in the previous form factor is a slotted circular channel [Fig. 4] due to the higher computational effort. For the sake of comparing the proposed solutions in a different form factor, comes the remaining geometries in $15.6 \mathrm{~mm}$ [Fig. 4 $\& 5]$.

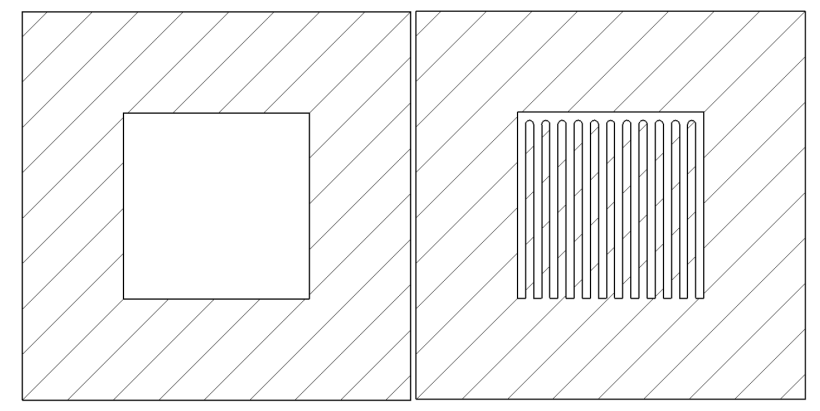

Figure 3- Square profiles.

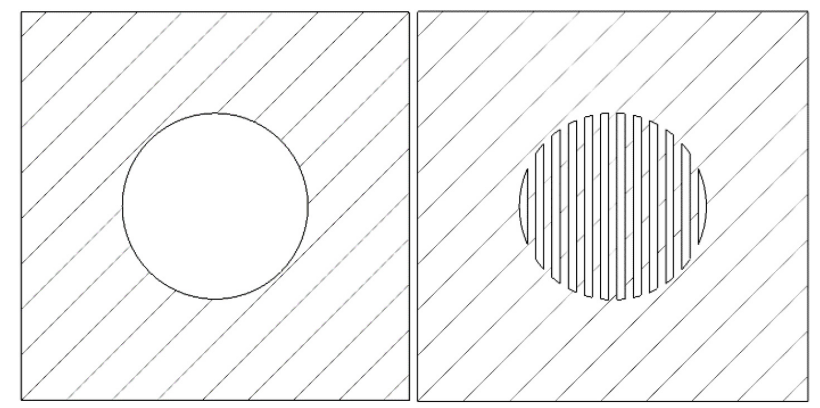

Figure 4- Circular and slotted profiles. 


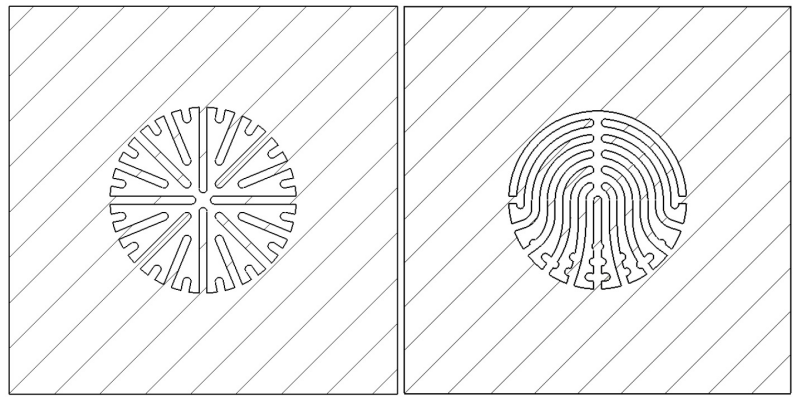

Figure 5- Profiles with fins.

\section{Test Methodology}

For the fins a design constraint of $0.2 \mathrm{~mm}$ minimum thickness [T] and space between fins was considered, which is close to the current capabilities of actual selective laser melting equipment [9].

The channels themselves have $4.6 \mathrm{~mm}$ in the first approach, later observations are made with channels of $15.8 \mathrm{~mm}$ in diameter [D] and ones of equal area respective to the original circular passage. Note that a generic $50 \mathrm{~mm}$ length [L] and proportional distance from the hot surface to the nearest point in the cooling gallery is kept constant throughout the simulations.

For the 4.6 form factor the side (S) is $9.8 \mathrm{~mm}$ and for 15.8 form factor is $33 \mathrm{~mm}$ [Fig. 6].
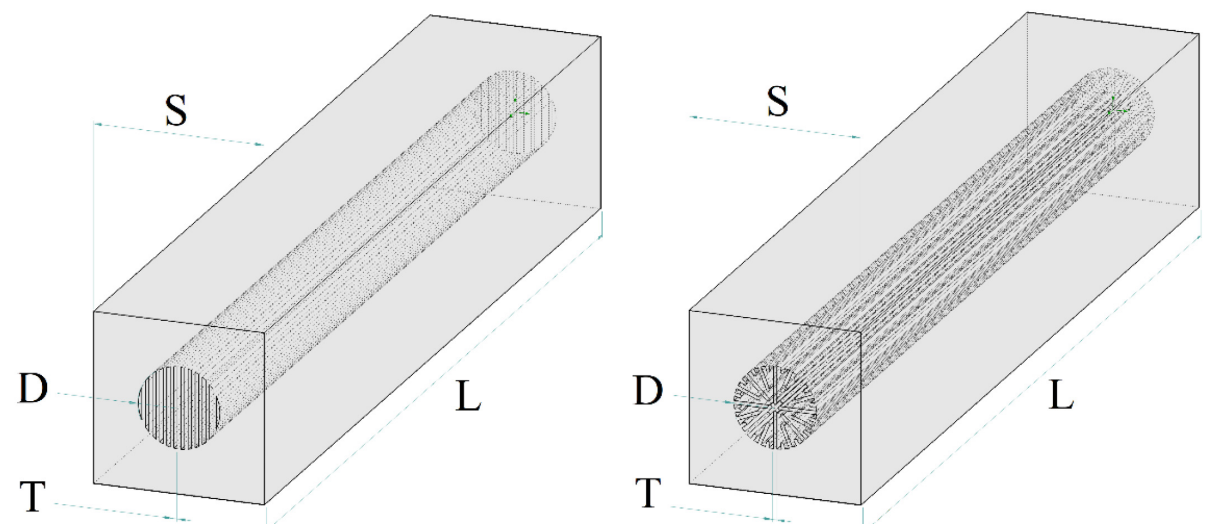

Figure 6- Representation of overall dimensions.

The test methodology consists in using a well-known CAD Flow Simulation tool in which a control volume is established to resemble each geometry to be tested. Then an outer wall temperature [W] of $200^{\circ} \mathrm{C}$ [10] and for the incoming flow, $20^{\circ} \mathrm{C}$ as for ambient temperature, a mass flow [I] of $0.7 \mathrm{~g} / \mathrm{s}$ [calibrated for the exit temperature to observe greater differences for the established length] for the smaller diameter, $8,3 \mathrm{~g} / \mathrm{s}$ [proportionally greater] for the larger and outlet $[\mathrm{P}]$ at atmospheric pressure of $101.3 \mathrm{kPa}$. Note that at the entry the flow is already fully developed to study only the turbulent flow [Fig. 7].
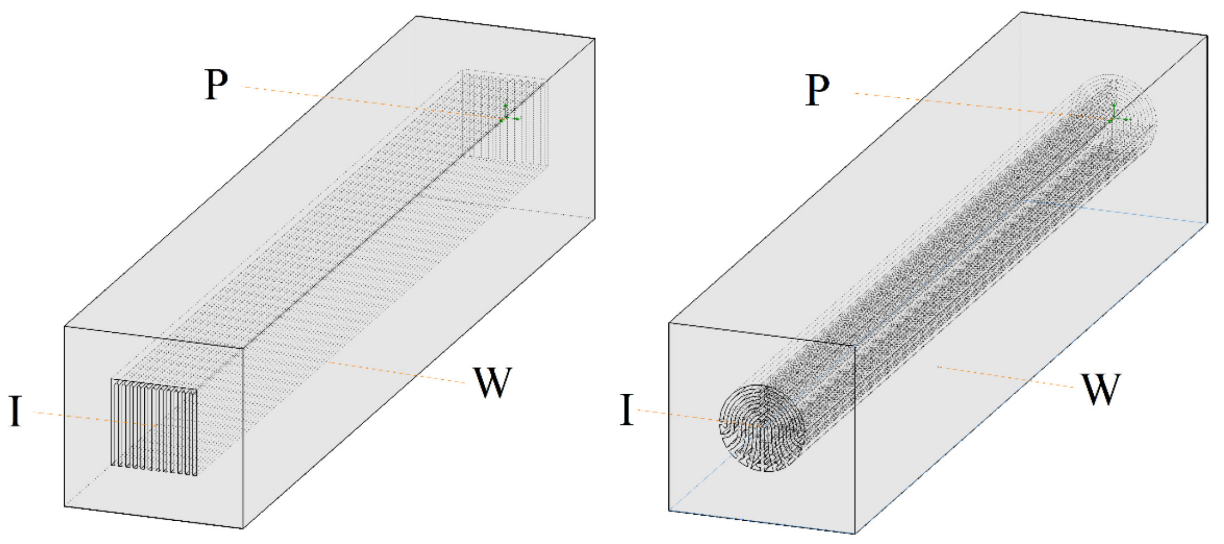

Figure 7- Representation of boundary conditions. 
On future studies larger scale models [Fig. 8] could be analyzed with the insertion of said fins, and be compared against conventional conformal designs. This way more practical results can be obtained
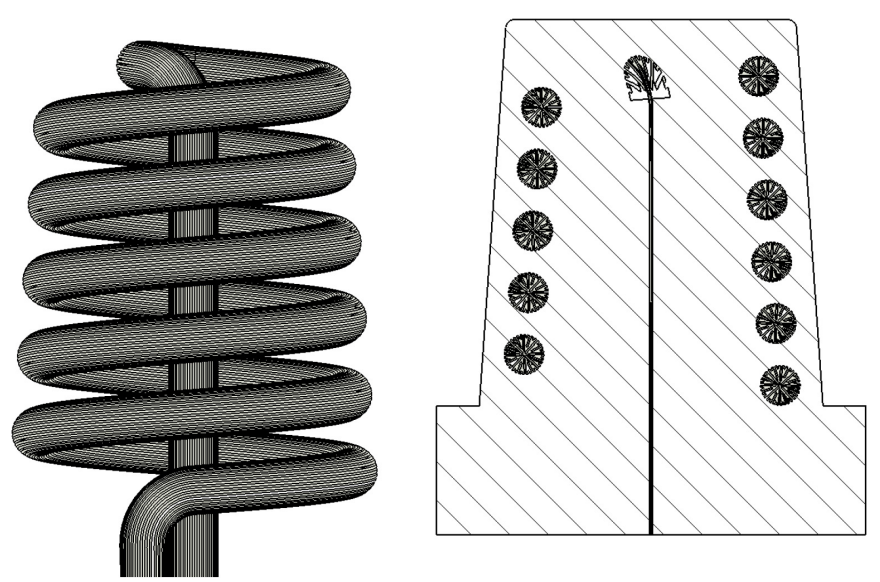

Figure 8 - Examples of application of conformal cooling channels and cross section.

\section{Flow Simulation Model}

The Flow Simulation tool applied is adequate to test ideas and conceptual design, it is another take on the Navier-Stokes equations that all the other CFD tools. On this case Flow Simulation allows for a Multiphysics solver in which flow analysis calculations are conducted in the fluid region and heat transfer and direct current calculations on solid regions respectively [11].

The mesh generation for the CAD geometry in this case preferentially follows a Cartesian-based mesh in which hexahedral cuboid elements are generated where the geometric boundaries do not intersect [Fig. 9] and if they were to bypass such boundaries, the elements are redesigned to accommodate the geometric contours [11] [Fig. 10].

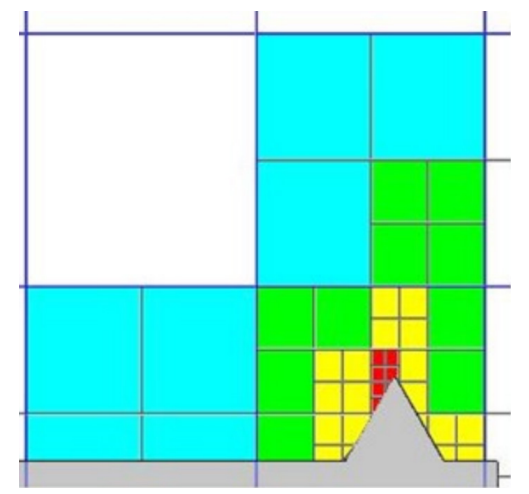

Figure 9- Cartesian based mesh [11]. 

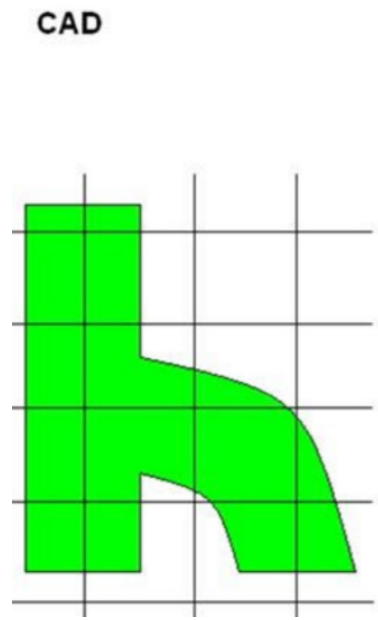
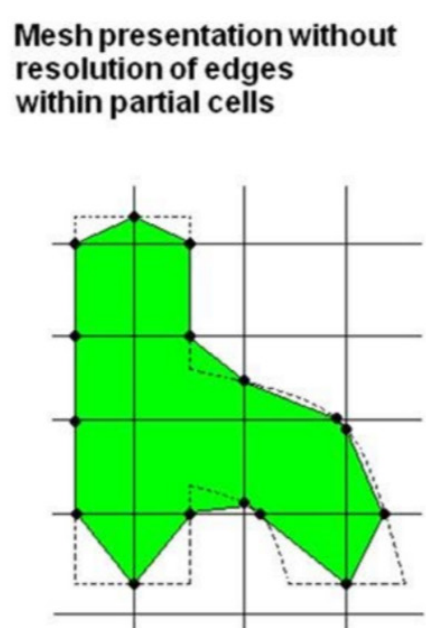

Mesh presentation with resolution of edges within partial cells

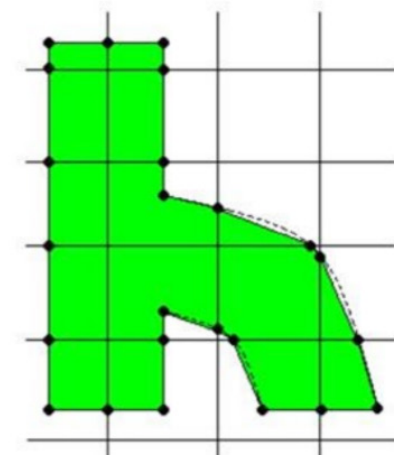

Figure 10- Accommodation of geometric contours [11].

\section{Results Convergence}

Before the main analysis of this paper could begin another had to be made, regarding the convergence of results affected by the meshing parameters. For this the channel with the directional fins with the form factor of $4,8 \mathrm{~mm}$ was used because it's features the most complex shape and a smaller overall cross-section compared to the $15,8 \mathrm{~mm}$ counterpart, for this element's minimum edge size has to be inferior to $0,2 \mathrm{~mm}$.

Table 1- Convergence for number of elements.

\begin{tabular}{lll}
\hline $\mathrm{N}^{\mathrm{o}}$ of elements & Heat rate $(\mathrm{W})$ & Difference $(\%)$ \\
\hline 384 & 230,5 & 8,84 \\
1224 & 226,3 & 6,85 \\
5000 & 219,4 & 3,59 \\
13720 & 212,7 & 0,42 \\
39200 & 213,9 & 0,97 \\
108192 & 212,3 & 0,22 \\
316800 & 211,8 & 0 \\
\hline
\end{tabular}

For this preliminary approach about conformal cooling, present in Table 1, a $0,5 \%$ criteria for convergence was applied, and it was found, after factoring the solver's time, that around one hundred thousand elements the criteria are met without the oscillation found between 13720 and 39200 elements.

Note that the remainder of results will only present one decimal place since that's what the $0.5 \%$ criteria allows to accurately represent, and that this value for the criteria was chosen empirically given the order of magnitude of the output values.

\section{Results for the 4,6mm Form Factor}

For this comparison an outer diameter was kept unchanged between compared geometries, and for the same mass flow this equates to different velocity profiles, this would still be verified if the same area is established since the hydraulic diameter varies with the duct's shape, this though could not be determined through For many in the mould industry or in thermal machines design the size of the application is critical, or on this case a heat carrying structure, so an overall size constraint was prioritized. 
Table 2- 4.6 form factor.

\begin{tabular}{|c|c|c|c|c|c|c|}
\hline Geometry & $\begin{array}{l}\text { Feature size } \\
(\mathrm{mm})\end{array}$ & $\begin{array}{l}\text { Diameter } \\
(\mathrm{mm})\end{array}$ & $\begin{array}{l}\text { Mass flow } \\
(\mathrm{g} / \mathrm{s})\end{array}$ & $\begin{array}{l}\text { Aspect } \\
\text { ratio }\end{array}$ & $\begin{array}{l}\text { Temperature } \\
\left({ }^{\circ} \mathrm{C}\right)\end{array}$ & $\begin{array}{l}\text { Heat rate } \\
\text { (W) }\end{array}$ \\
\hline Simple & \multirow[t]{2}{*}{--- } & \multirow{6}{*}{4,6} & \multirow{6}{*}{0,7} & 0,9 & 71,4 & 97,1 \\
\hline Radial fins & & & & 7,4 & 88,5 & 199,8 \\
\hline Slotted & \multirow{4}{*}{0,2} & & & 10,9 & 88,4 & 210,5 \\
\hline Directional & & & & 57,5 & 89,6 & 212,3 \\
\hline Squared & & & & 0,9 & 74,2 & 99,4 \\
\hline Square fins & & & & 9,9 & 91,9 & 217,3 \\
\hline
\end{tabular}

These tests find that the inclusion of fins within a circular pipe, yields better results for the established conditions, this is observable by the fluid's exiting temperature. Though this may not always be the case, since the flow characteristics like turbulence, affects the heat transfer coefficient, for this the heat rate is observed.

From the tested geometries the radial slots were the least effective relative to the original design, achieving a 140\% improvement in the heat transfer rate, and it's in a 5\% range from the best results.

Both the slotted design and the directional fins are in close margin temperature and heat rate wise despite the directional design having a greater aspect ratio [5.3 times], which is given by the proportion of the shape's overall perimeter and its area, so as there is a point of diminishing returns with the increase of Reynolds number [12], here the same is verified for the aspect ratio for the previously stated flow conditions.

As this could be considered an alternative to the current topological methods, it should be stated that the simulation time is greatly affected by the increase of complexity by the means of the number of elements of a certain size that are required to recreate any given geometry in the computational domain. And the slotted design not being the most complex is the one that required up to five times more processing time in these simulations.

It's also interesting to see how heat is being transferred form the hot surface to the cooling fluid, the fins also show a much higher dissipation rate at their base, but overall is more evenly distributed, which could reduce the occurrence of hot spots in the moulding surface [Fig. 11]. 

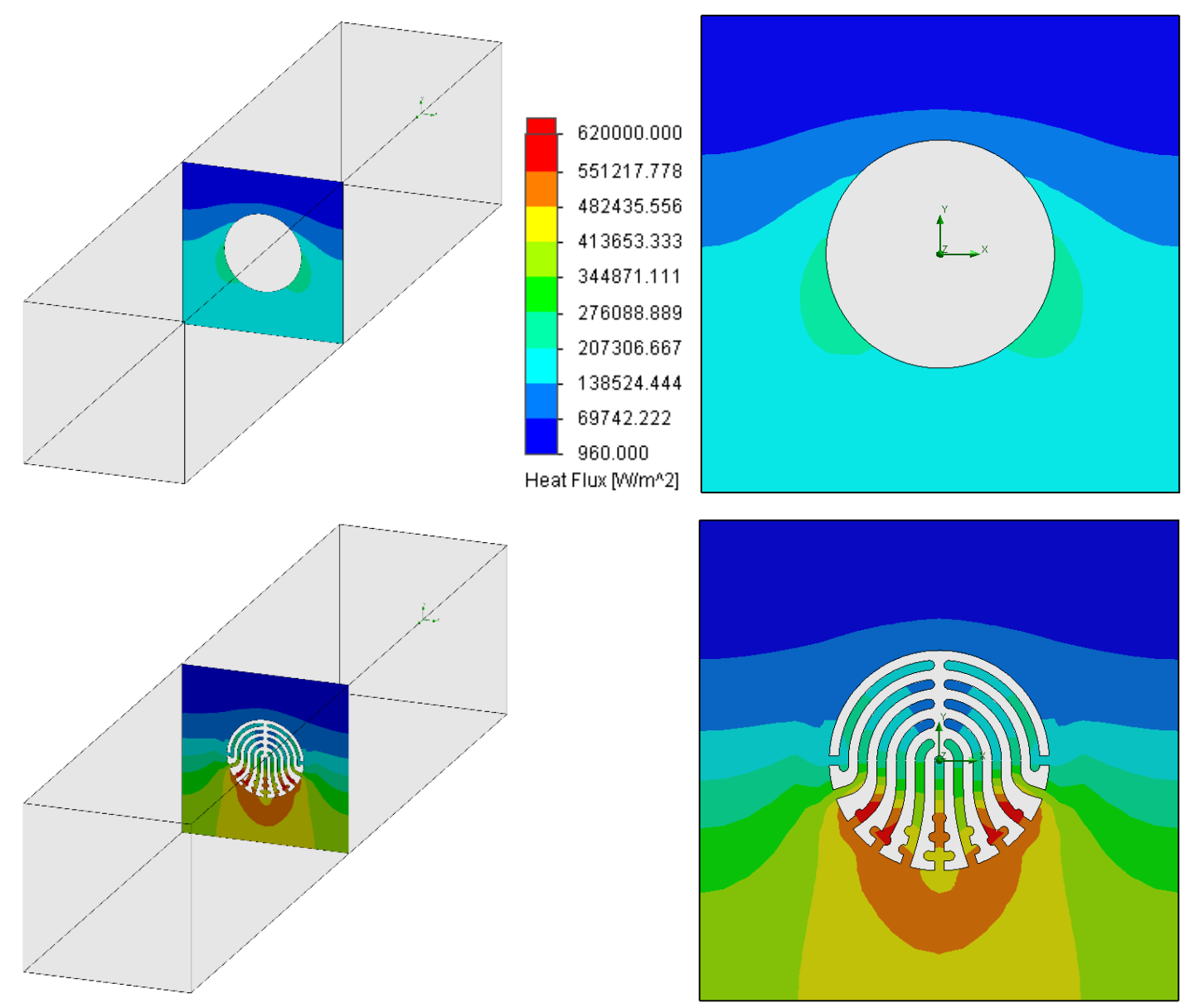

Figure 11- Heat flux plots at the middle of the part for the simple and directionally finned design

Despite the goals that were set around the circular profiles for these simulations, it is interesting to observe that the same constructive principles apply to other geometries like square passages showing improvements of the same order of magnitude [119\% higher heat transfer].

\section{Results for the $\mathbf{1 5 , 8 m m}$ Form Factor}

A second approach was taken to help understand what kind of scaling can be had with the same constructive constraints of 0.2 millimeters and a mass flow proportionally bigger to the area increase of the simple circular solution. On this take the directional geometry was used since the computational effort was lower and to test a different iteration of the same design in larger scale.

Table 3- 15.8 form factor.

\begin{tabular}{lllllll}
\hline Geometry & $\begin{array}{l}\text { Feature } \\
\text { size } \\
(\mathrm{mm})\end{array}$ & $\begin{array}{l}\text { Diameter } \\
(\mathrm{mm})\end{array}$ & $\begin{array}{l}\text { Mass } \\
\text { flow } \\
(\mathrm{g} / \mathrm{s})\end{array}$ & $\begin{array}{l}\text { Aspect } \\
\text { ratio }\end{array}$ & $\begin{array}{l}\text { Temperature } \\
\left({ }^{\circ} \mathrm{C}\right)\end{array}$ & $\begin{array}{l}\text { Heat } \\
\text { rate } \\
(\mathrm{W})\end{array}$ \\
\hline Simple & --- & 15.8 & 8.3 & 0.3 & 33.1 & 172.9 \\
Directional & 0.2 & & & 8.6 & 28.4 & 351.0 \\
\hline
\end{tabular}

For this increase in diameter and the dimensional constraint, the aspect ratio is much lower, and thus affects the heat rate capacity. Making this geometry almost two times as better as the circular channel instead of $125 \%$ as previously observed.

Another point of interest comes in the form of construction constraints in which it was considered that the minimal feature size could be anything from the prior established $0.2 \mathrm{~mm}$ and $0.6 \mathrm{~mm}$, with $0.1 \mathrm{~mm}$ of scaling between each test as presented in Fig. 12. On this case the radially slotted geometry was used because of its scalability. With this it's expected to establish some relation amongst parameters and results. 


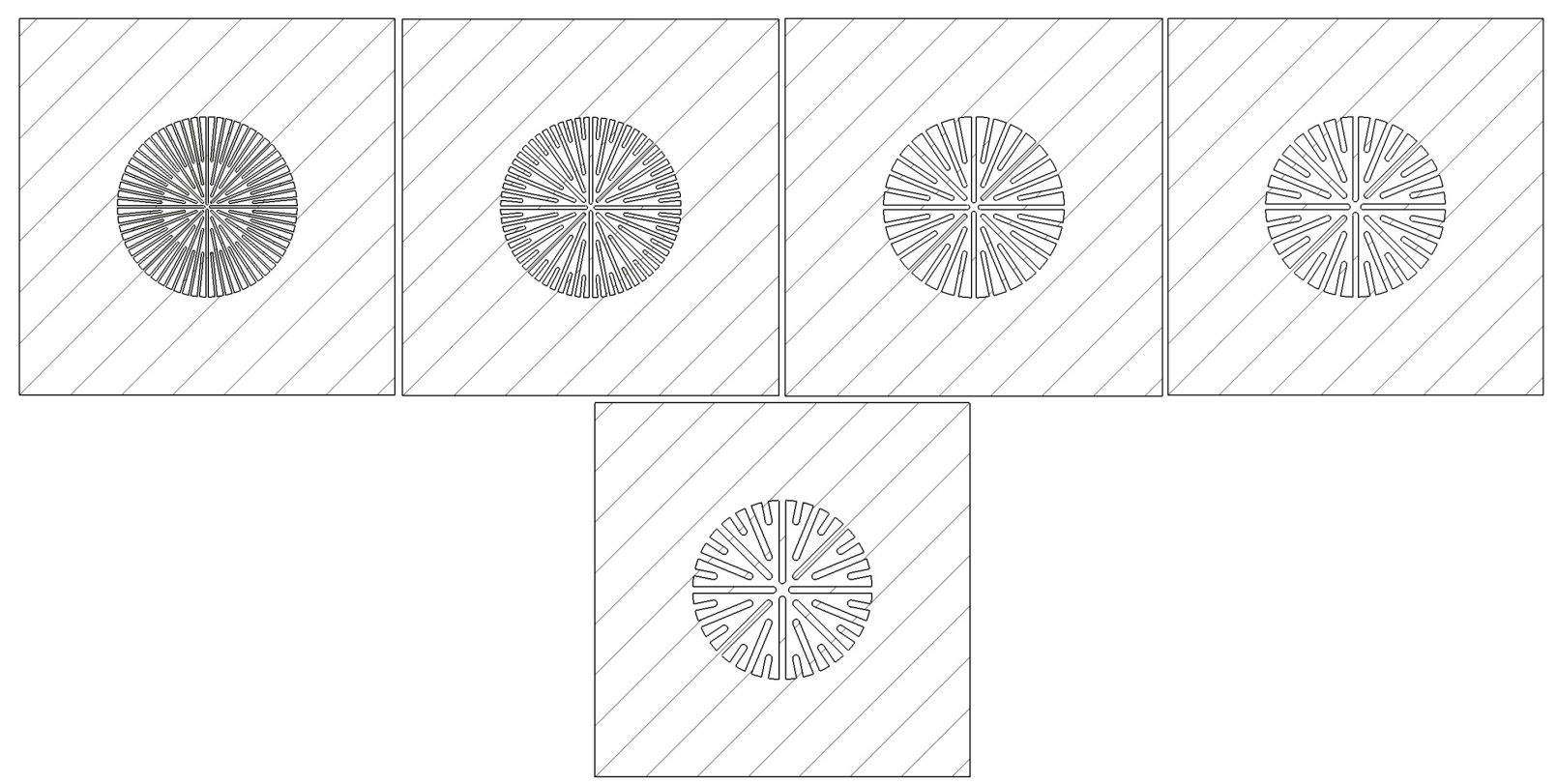

Figure 12- Profile with radial fins of different thickness [0.2mm to $0.6 \mathrm{~mm}]$.

Table 4- Radial fins with varying feature thickness.

\begin{tabular}{lllllll}
\hline Geometry & $\begin{array}{l}\text { Feature } \\
\text { size } \\
(\mathrm{mm})\end{array}$ & $\begin{array}{l}\text { Diameter } \\
(\mathrm{mm})\end{array}$ & $\begin{array}{l}\text { Mass } \\
\text { flow } \\
(\mathrm{g} / \mathrm{s})\end{array}$ & $\begin{array}{l}\text { Aspect } \\
\text { ratio }\end{array}$ & $\begin{array}{l}\text { Temperature } \\
\left({ }^{\circ} \mathrm{C}\right)\end{array}$ & $\begin{array}{l}\text { Heat } \\
\text { rate } \\
(\mathrm{W})\end{array}$ \\
\hline \multirow{5}{*}{ Radial fins } & 0,2 & & & 5,6 & 28,6 & 339,4 \\
& 0,3 & & & 4,5 & 28,4 & 324,1 \\
& 0,4 & 15,8 & 8,3 & 3,0 & 28,5 & 310,8 \\
& 0,5 & & & 2,8 & 28,8 & 308,3 \\
& 0,6 & & & 2,5 & 29,1 & 306,4 \\
\hline
\end{tabular}

With the increase of the feature size comes, naturally with this geometry, an aspect ratio decrement which is correlating to the heat rate of the sample but not necessarily the outlet temperature, so that for this case a drop in the thermal conductivity coefficient can be inferred from its defining equation [Eq. 1].

$$
h=\frac{q}{\Delta \mathrm{T}}
$$

Equation 1- Heat transfer coefficient.

The take away from the aforementioned results is that the feature size [the lower the better] that any given process permits dictates the heat rate potential for the specified flow conditions. Likewise the orientation of the populating fins affects this heat rate. And that in spite of a higher aspect ratio promoting a better heat transfer capacity, there's a clear point of diminishing returns that can be quickly offset by the system's pressure.

\section{Mould Cooling Project Methodology}

As the results have shown, the inclusion of thin fin-like structures inside a heat carrying channel proves to be beneficial, up to 2.4 times the cooling capacity [best case scenario Table 2]. For this it's worth to elaborate a concise project guide to aid the implementation of this type of solution. Although it should be noted that this is a preliminary approach in which some assumptions will take place, regarding the following developments of this study [Fig. 13]. Note that the channels are considered for both heating and cooling. 


\section{CAD}

Models of the mold core, cavity and part

Materials selection
Mould and manufacturing method
Plastic Injection Simulation
Output: Temperature and Pressure profile
Project Conformal Channels
Empirical placement
Choose Fin Geometry proximity between the channels
From library to populate the inner
surface of the channels

Figure 13- Flowchart of mould project methodology

\section{Conclusions}

The current conformal cooling strategies that additive manufacturing enables still show a wide margin for improvement as far as empiric design allows. This study shows that populating the internal geometry of a cooling channel with fins growing from the direction of the heat source, yields significant gains for a low requirement in computational power, furthermore this solution can be embedded within a CAD software as a tool that populates a control volume following predefined construction constraints and a geometrical pattern that is best suited for the flow conditions imposed by the user.

A point of interest for future studies would be the implementation of Wick-like structures like the ones found in heat pipes featuring a high surface area to volume ratio, which can displace heat through the phase change of a fluid enabled by the capillarity of the structure. Although care should be taken, because a structure like this is well beyond the point of diminishing returns regarding the aspect ratio, and could be in a region where the pressure drop along the channel could prohibit the implementation. 


\section{Acknowledgements}

This work was supported by the Portuguese Foundation for Science and Technology (FCT) through the following project: UID/Multi/04044/2013 and PAMI - ROTEIRO/0328/2013 (N ${ }^{\circ}$ 022158). In addition, the authors acknowledge the funding from the project Coolgrafeno, copromoção n. 17883 from the Portuguese National Innovation Agency.

\section{References}

[1] A. J. V. Pontes, SHRINKAGE AND EJECTION FORCES IN INJECTION MOULDED PRODUCTS, Minho, 2002.

[2] J. MALLON, "Advances in Automation for Plastics Injection Moulding," Shropshire, 2001.

[3] J. Williams, "Injection Molding Cooling Time: A Breakdown".

[4] V. G, R. K. Y and R. G, "Comparison of Straight Line to Conformal Cooling Channel in Injection Molding," materialstoday: PROCEDINGS, vol. 4, 2017.

[5] R.-Y. Zhang, W. Yang, J.-J. Wu, J. Feng, J.-M. Feng, B.-H. Xie and M.-B. Yang, "Hierarchically oriented crystalline structures of HDPE induced by strong second melt penetration," The Royal Society of Chemistry, 2014.

[6] Autodesk, "The Causes of Warpage," [Online]. Available: https://www.autodesk.com/industry/ manufacturing/resources/injection-molding/causes-of-warpage.

[7] B. Clark, "Fundamentals of Designing the Optimal Cooling System," MoldMaking Technology, 1212014.

[8] K. Yaji, T. Yamad, S. Kubo, K. Izui and S. Nishiwaki, "A topology optimization method for a coupled thermal-fluid problem using level set boundary expressions," International Journal of Heat and Mass Transfer, vol. 81, pp. 878-888, 2015.

[9] SLM-Solutions, "Selective Laser Melting Machine SLM 500," [Online]. Available: https://slmsolutions.com/products/machines/selective-laser-melting-machine-slm-500.

[10] INEOS Olefins \& Polymers USA, "Polypropylene Processing Guide," 2007. [Online]. Available: https://www.ineos.com/globalassets/ineos-group/businesses/ineos-olefins-andpolymers-usa/products/technical-information--

patents/ineos_polypropylene_processing_guide.pdf.

[11] A. Sobashkin and G. Dumnov, "Numerical Basis of CAD-Embedded CFD," February 2014. [Online].

[12] HRS heat exchangers, "Comparison of Laminar and Turbulent Flow," 17 August 2016. [Online]. Available: https://www.hrs-heatexchangers.com/resource/comparison-laminarturbulent-flow/. 